\title{
Correction to: Dietary antioxidative supplements and diabetic retinopathy; a systematic review
}

\author{
Ozra Tabatabaei-Malazy ${ }^{1,2} \cdot$ Edris Ardeshirlarijani $^{3} \cdot$ Nazli Namazi $^{4} \cdot$ Shekoufeh Nikfar ${ }^{2,5,6,7} \cdot$ Reza Baradar Jalili $^{8}$. \\ Bagher Larijani $^{2}$
}

Published online: 5 November 2019

(C) Springer Nature Switzerland AG 2019

\section{Correction to: Journal of Diabetes \& Metabolic Disorders. https://doi.org/10.1007/s40200-019-00434-x}

Please note the following corrections to the original article.

The 2nd author affiliation should read Endocrinology and Metabolism Research Center, Endocrinology and Metabolism Clinical Sciences Institute, Tehran University of Medical Sciences, Tehran, Iran.

The 3rd author affiliation should read Simo Fraser University, Burnaby, Canada.

Corrections in Tables 1 and 2 as follows:

1- in t1.10: Lam et al.: please move up these words " $r=0.33$ ";

2- The first "I" in "lipid profile" line t1.17 should be capital.
3 "ARIC study and its expansion" has been deleted from footnote of Table 1.

4 In 2.4 it should read Se and not sel.

In the Introduction the first line should read 'Diabetic retinopathy (DR) that is definded as retinal micro-vascular lesion in subjects with diabetes classified...'

The original article has been corrected'.

Publisher's note Springer Nature remains neutral with regard to jurisdictional claims in published maps and institutional affiliations.
The online version of the original article can be found at https://doi.org/ 10.1007/s40200-019-00434-x

Bagher Larijani

emrc@tums.ac.ir

Ozra Tabatabaei-Malazy

tabatabaeiml@sina.tums.ac.ir

Edris Ardeshirlarijani

ediardeshir@gmail.com

Nazli Namazi

nazli.namazi@yahoo.com

Shekoufeh Nikfar

shekoufeh.nikfar@gmail.com

Reza Baradar Jalili

reza.jalili@ubc.ca

1 Non-Communicable Diseases Research Center, Endocrinology and Metabolism Population Sciences Institute, Tehran University of Medical Sciences, Tehran, Iran
2 Endocrinology and Metabolism Research Center, Endocrinology and Metabolism Clinical Sciences Institute, Tehran University of Medical Sciences, Tehran, Iran

3 Simo Fraser University, Burnaby, Canada

4 Diabetes Research Center, Endocrinology and Metabolism Clinical Sciences Institute, Tehran University of Medical Sciences, Tehran, Iran

5 Personalized Medicine Research Center, Endocrinology and Metabolism Clinical Sciences Institute, Tehran University of Medical Sciences, Tehran, Iran

6 Evidence-based Evaluation of Cost-Effectiveness and Clinical Outcomes Group, The Institute of Pharmaceutical Sciences (TIPS), Tehran University of Medical Sciences, Tehran, Iran

7 Department of Pharmacoeconomics and Pharmaceutical Administration, Faculty of Pharmacy and Pharmaceutical Policy Research Center, Tehran University of Medical Sciences, Tehran, Iran

8 Department of Surgery, University of British Columbia, Vancouver, British Columbia, Canada 